\title{
Cross-Hedging with Currency Options and Futures
}

\author{
Eric C. Chang and Kit Pong Wong*
}

\begin{abstract}
This paper develops an expected utility model of a multinational firm facing exchange rate risk exposure to a foreign currency cash flow. Currency derivative markets do not exist between the domestic and foreign currencies. There are, however, currency futures and options markets between the domestic currency and a third currency to which the firm has access. Since a triangular parity condition holds among these three currencies, the available, yet incomplete, currency futures and options markets still provide a useful avenue for the firm to indirectly hedge against its foreign exchange risk exposure. This paper offers analytical insights into the optimal cross-hedging strategies of the firm. In particular, the results show the optimality of using options in conjunction with futures in the case of currency mismatching, even though cash flows appear to be linear.
\end{abstract}

\section{Introduction}

Since the collapse of the Bretton Woods Agreement in 1973, exchange rates have become substantially volatile (Meese (1990)). In response to increased exchange rate fluctuations and to various accounting rules and regulations, ${ }^{1}$ multinational firms take foreign exchange risk management very seriously (Rawls and Smithson (1990)). As documented by Bodnar, Hayt, and Marston (1996), (1998) and Bodnar and Gebhardt (1999), the use of currency forwards, futures, and options is the norm rather than the exception in modern corporations.

Despite the widespread use of currency options in foreign exchange risk management, the question concerning their proper application as a hedging in-

*Chang, ecchang@business.hku.hk, School of Business, and Wong, kpwong@econ.hku.hk, School of Economics and Finance, University of Hong Kong, Pokfulam Road, Hong Kong. We thank Nicole Branger, Rafi Eldor, Ron Giammarino, Jonathan Karpoff (the editor), Donald Lien, Harris Schlesinger, Clifford Smith, Vasant Naik (associate editor and referee), and seminar participants at the University of Hong Kong, the 2000 Georgia Tech/Fortis International Finance Conference at Georgia Tech, and the 2001 German Finance Association Conference in Vienna for helpful comments and suggestions. We also gratefully acknowledge financial support from the Center for Financial Innovation and Risk Management (CFIRM) at the University of Hong Kong and from research grants provided by the Research Grant Council of the Hong Kong government.

${ }^{1}$ The Financial Accounting Standards Board requires transaction and translation gains and losses to be reported in income statements. The Securities and Exchange Commission, on the other hand, requires statements explaining the impact of exchange rate movements on reported profits to be included in annual reports. 
strument remains largely unexplored. ${ }^{2}$ The purpose of this paper is to examine the hedging role of currency options in the context of cross-hedging. While currency derivative markets for forwards, futures, and options are the hallmark of industrialized countries, they are seldom readily available in less developed countries (LDCs) in which capital markets are embryonic and foreign exchange markets are heavily controlled. ${ }^{3}$ Also, in many of the newly industrializing countries of Latin America and Asia Pacific, currency derivative markets are just starting to develop at a rather slow pace. ${ }^{4}$ Multinational firms exposed to currencies of these countries thus have to avail themselves of derivative securities on related currencies to cross-hedge against their foreign exchange risk exposure.

This paper offers analytical insights into the optimal cross-hedging strategies of a multinational firm facing exchange rate risk exposure to a foreign currency cash flow. We investigate a scenario in which currency derivative markets do not exist between the domestic and foreign currencies. There are, however, currency futures and options markets between the domestic currency and a third currency to which the firm has access. Since a triangular parity condition holds among these three currencies, cross-hedging provided by the available, yet incomplete, currency futures and options markets is useful to the firm in reducing its foreign exchange risk exposure. Our results demonstrate the optimality of using options in conjunction with futures in the case of currency mismatching, even though cash flows appear to be linear. One driving force is the triangular parity condition, which gives rise to an exchange rate risk that is multiplicative, thereby non-linear, in nature. This source of non-linearity creates a hedging demand for non-linear payoff currency options distinct from that for linear payoff currency futures.

Under two rather restrictive conditions, namely, i) quadratic utility functions and ii) independent spot exchange rates, we show that currency options play no role as a hedging instrument. Given unbiased futures and options prices, it is well known that the firm should choose a cross-hedge position that minimizes the variability of the marginal utility across different realizations of the spot exchange rate between the domestic and third currencies. Condition i) ensures that the firm's marginal utility function is linear, while condition ii) ensures that the firm's foreign exchange risk exposure is linear. It thus follows immediately from conditions $i$ and ii that the firm's optimal cross-hedge position should contain no currency options. However, if either condition is violated, non-linearity is present in the variability of the firm's marginal utility across different realizations of the spot exchange rate. These two sources of non-linearity induce the firm to use non-linear payoff currency options for hedging purposes. Thus, cross-hedging provides a rationale for the hedging role of currency options.

\footnotetext{
${ }^{2}$ Indeed, as shown by Benninga and Blume (1985), investors in the Black-Scholes (1973) world would purchase options only when their utility functions exhibit bizarre properties, which is highly unlikely. See also Leland (1980), Brennan and Solanki (1981), and Franke, Stapleton, and Subrahmanyam (1998) for the demand for options in the setting of portfolio insurance.

${ }^{3}$ Even if currency forward contracts are available in some LDCs, they are deemed to be forwardcover insurance schemes rather than financial instruments whose prices are freely determined by market forces (Jacque (1996)).

${ }^{4}$ See Eiteman, Stonehill, and Moffett (1998) for a description of the currency regime and the status of currency derivatives in many of these so-called "exotic currencies."
} 
The rest of the paper is organized as follows. In the next section, we develop a static model of a multinational firm facing multiple currency risks and crosshedging opportunities. Section III derives the firm's optimal cross-hedge position when unbiased currency futures and options markets between the domestic currency and a third currency are present. Section IV extends the static model into a dynamic one and derives the firm's optimal dynamic cross-hedge positions. Section $\mathrm{V}$ performs an empirical exercise to show the merits of including currency futures and options for cross-hedging purposes. The final section concludes.

\section{The Model}

Consider a one-period, two-date ( 0 and 1$)$ model of a multinational firm that has an operation domiciled in a foreign country. At date 1 , the firm receives a net cash flow, $X$, from its foreign operation, where $X$ is denominated in the foreign currency. While the firm knows with certainty the size of $X$ at date 0 , it does not know the then prevailing spot exchange rate at date $1, \tilde{S}$, which is expressed in units of the domestic currency per unit of the foreign currency. ${ }^{5}$ The firm as such encounters foreign exchange risk exposure of $\tilde{S} X$.

There are no currency derivative markets between the domestic and foreign currencies. The firm, however, has access to currency futures and options markets between the domestic currency and a third currency. Define $\tilde{S}_{1}$ as the date 1 spot exchange rate of the domestic currency against the third currency. Likewise, define $\tilde{S}_{2}$ as the date 1 spot exchange rate of the third currency against the foreign currency. Based on these two spot exchange rates, one can derive a date 1 crossrate of the domestic currency against the foreign currency as $\tilde{S}_{1} \tilde{S}_{2}$. It follows immediately from the law of one price that $\tilde{S}=\tilde{S}_{1} \tilde{S}_{2}$. Such a triangular parity condition is depicted in Figure 1.

FIGURE 1

Triangular Parity Condition

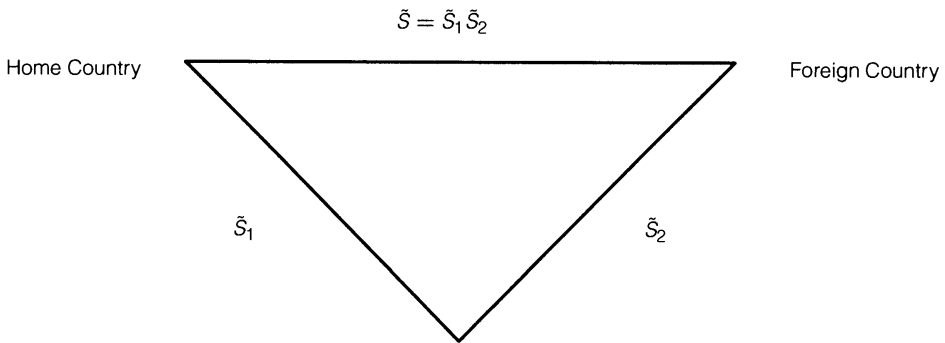

Third Country

For ease of exposition, we write

$$
\tilde{S}_{1}=\bar{S}_{1}+\tilde{\theta}
$$

\footnotetext{
${ }^{5}$ Throughout the paper, random variables have a tilde $(\sim)$ while their realizations do not.
} 
where $\bar{S}_{1}$ is the expected value of $\tilde{S}_{1}$, and $\tilde{\theta}$ is a zero mean, finite variance random variable. Furthermore, we consider a linear projection of $\tilde{S}_{2}$ on $\tilde{S}_{1}$ to yield

$$
\tilde{S}_{2}=\bar{S}_{2}+\beta\left(\tilde{S}_{1}-\bar{S}_{1}\right)+\tilde{\epsilon}=\bar{S}_{2}+\beta \tilde{\theta}+\tilde{\epsilon},
$$

where $\bar{S}_{2}$ is the expected value of $\tilde{S}_{2}, \beta$ is a constant, $\tilde{\epsilon}$ is a zero mean, finite variance random variable independent of $\tilde{\theta}$, and the second equality follows from equation (1). If $\beta$ is negative (positive), $\tilde{S}_{1}$ and $\tilde{S}_{2}$ are negatively (positively) correlated. These two random variables are independent only when $\beta=0 .{ }^{6}$

If the firm is privately held, the owners' assets may be concentrated in the firm and thus risk-averse behavior prevails. If the firm is publicly listed, standard finance theory suggests that shareholders of the firm should be de facto risk neutral given their ability to diversify in well-functioning capital markets. Agency theory, however, recognizes that there is separation of ownership and control in modern corporations. Managers who run the firm are averse to the risk that a sequence of losses could lead to dismissal, and thus the decisions made by the firm may also reflect risk aversion (Stulz (1984)). ${ }^{7}$ In either case, we assume that the firm's date 1 domestic currency income is mapped onto utility through a strictly increasing and concave function, $U$, indicating the presence of risk aversion. ${ }^{8}$

To hedge against its foreign exchange risk exposure, the firm can trade infinitely divisible currency futures and options (calls and puts), which call for delivery of the domestic currency per unit of the third currency at date 1. Because payoffs of any combinations of futures, calls, and puts can be replicated by any two of these three financial instruments (Sercu and Uppal (1995)), one of them is redundant. It is therefore no loss of generality to restrict the firm to use currency futures and put options only. Furthermore, purely for the sake of simplicity, we consider only one strike price for the currency put options, which is set equal to $\bar{S}_{1}$. Let $P$ be the premium on the currency put options, where $P$ is compounded to date 1.

The firm's date 1 income denominated in the domestic currency is given by

$$
\tilde{\Pi}=\tilde{S}_{1} \tilde{S}_{2} X+\left(F-\tilde{S}_{1}\right) H+\left[P-\max \left(\bar{S}_{1}-\tilde{S}_{1}, 0\right)\right] Z,
$$

where $F$ is the date 0 futures price, and $H$ and $Z$ are the numbers of the currency futures and put options sold (purchased if negative) at date 0 , respectively. The firm is an expected utility maximizer and has to solve the following ex ante decision problem,

$$
\max _{H, Z} E[U(\tilde{\Pi})]
$$

\footnotetext{
${ }^{6}$ Intuitively, if the economy of the third country is strong (weak), we would expect that one unit of the third currency should exchange for more (less) units of the domestic and foreign currencies, ceteris paribus. In other words, $S_{1}$ should go up (down) while $S_{2}$ should go down (up) when the economy of the third country is strong (weak). It is thus reasonable to believe that $\beta<0$ in general. See Table 1 for empirical evidence.

${ }^{7}$ Other rationales for corporate hedging include taxes (Smith and Stulz (1985)), costs of financial distress (Smith and Stulz (1985)), and capital market imperfections (Stulz (1990), Froot, Scharfstein, and Stein (1993)). In all these cases, a firm's profit function can always be modeled as a concave function. In this regard, our assumption of risk aversion can be viewed as an approximation of the firm's true objective function.

${ }^{8}$ See Tufano (1996) for evidence that managerial risk aversion is a relevant rationale for corporate risk management in the gold mining industry.
} 
where $E$ is the expectation operator with respect to the firm's subjective joint probability distribution function of $\tilde{S}_{1}$ and $\tilde{S}_{2}$, and $\tilde{\Pi}$ is defined in equation (3).

\section{Optimal Cross-Hedging}

The first-order conditions for program (4) are given by

$$
\begin{aligned}
E\left[U^{\prime}\left(\tilde{\Pi}^{*}\right)\left(F-\tilde{S}_{1}\right)\right] & =0, \\
E\left\{U^{\prime}\left(\tilde{\Pi}^{*}\right)\left[P-\max \left(\bar{S}_{1}-\tilde{S}_{1}, 0\right)\right]\right\} & =0,
\end{aligned}
$$

where an asterisk $\left({ }^{*}\right)$ indicates an optimal level. The second-order conditions for a maximum are satisfied given risk aversion.

The firm's optimal cross-hedge position, $\left(H^{*}, Z^{*}\right)$, is implicitly defined in equations (5) and (6). Among other things, $\left(H^{*}, Z^{*}\right)$ would depend on whether the firm perceives the date 0 futures price and put option premium as unbiased or biased. In the former unbiased case, $\left(H^{*}, Z^{*}\right)$ should reflect solely the hedging motive of the firm. In the latter biased case, $\left(H^{*}, Z^{*}\right)$ reflects also the speculative motive of the firm. Since our objective is to show the hedging role of the currency futures and put options, we shall hereafter assume that the date 0 futures price and put option premium are perceived as unbiased by the firm. That is, $F=\bar{S}_{1}$ and $P=E\left[\max \left(\bar{S}_{1}-\tilde{S}_{1}, 0\right)\right] .^{9}$

\section{A. Redundancy of Options}

Given the joint unbiasedness of the date 0 futures price and put option premium, it follows from equations (1) and (2) that equation (3) is reduced to

$$
\tilde{\Pi}=\left(\bar{S}_{1}+\tilde{\theta}\right)\left(\bar{S}_{2}+\beta \tilde{\theta}+\tilde{\epsilon}\right) X-\tilde{\theta} H+[P-\max (-\tilde{\theta}, 0)] Z
$$

Using the covariance operator, Cov, we can write equations (5) and (6) as ${ }^{10}$

$$
\begin{aligned}
\operatorname{Cov}\left[U^{\prime}\left(\tilde{\Pi}^{*}\right), \tilde{\theta}\right] & =0, \\
\operatorname{Cov}\left[U^{\prime}\left(\tilde{\Pi}^{*}\right), \max (-\tilde{\theta}, 0)\right] & =0 .
\end{aligned}
$$

The firm's utility function, $U$, is said to be quadratic if it takes the generic form: $U(\Pi)=\Pi-b \Pi^{2}$, where $b$ is a positive constant. Based on equations (8) and (9), the following proposition is invoked (all proofs of propositions are relegated to the Appendix).

Proposition 1. Suppose that the prices of the currency futures and put options between the domestic and third currencies are perceived as jointly unbiased by the multinational firm. If the firm's utility function is quadratic and if $\tilde{S}_{1}$ and $\tilde{S}_{2}$ are independent, then it is optimal for the firm to fully hedge via the currency futures, $H^{*}=\bar{S}_{2} X$, and not to use the currency put options, $Z^{*}=0$.

\footnotetext{
${ }^{9}$ Our intention here is not to impose an ad hoc option pricing theory but to focus on the hedging role of currency options.

${ }^{10}$ For any two random variables, $\tilde{X}$ and $\tilde{Y}, \operatorname{Cov}(\tilde{X}, \tilde{Y})=E(\tilde{X} \tilde{Y})-E(\tilde{X}) E(\tilde{Y})$.
} 
The intuition underlying Proposition 1 is as follows. Since covariances can be interpreted as marginal variances, equation (8) implies that the firm's optimal cross-hedge position, $\left(H^{*}, Z^{*}\right)$, minimizes the variance of the marginal utility across different realizations of $\tilde{\theta}$. Substituting $H=\bar{S}_{2} X$ and $Z=0$ into equation (7) yields the firm's date 1 domestic currency income as

$$
\tilde{\Pi}=\bar{S}_{1} \bar{S}_{2} X+\left(\bar{S}_{1}+\tilde{\theta}\right) \tilde{\epsilon} X
$$

since $\beta=0$. If the firm's utility function is quadratic (i.e., $U^{\prime \prime \prime}=0$ ), the marginal utility function is linear. The variability of the marginal utility across different realizations of $\tilde{\theta}$ as such comes entirely from the variability of the date 1 domestic currency income of the firm, which in turn comes from the second term on the right-hand side of equation (10). Since this term is independent of $\tilde{\theta},{ }^{11}$ the firm faces no residual risk that is hedgeable, rendering the optimality of choosing $H^{*}=$ $\bar{S}_{2} X$ and $Z^{*}=0$ by the firm.

A direct implication of Proposition 1 is that under quadratic utility functions and independent spot exchange rates currency options would be redundant for cross-hedging purposes. However, it is unduly restrictive to assume both conditions to hold. In other words, in a realistic cross-hedging environment, we would expect currency options to be an integral part of the optimal hedge positions of multinational firms.

\section{B. Hedging Role of Options}

Consider the following simple example. Suppose that $\tilde{\epsilon} \equiv 0$. Furthermore, assume that $\tilde{\theta}$ takes on three possible values: $-\Theta$ with probability $p, 0$ with probability $1-2 p$, and $\Theta$ with probability $p$. Then, from equations (1) and (2) we have

$$
\tilde{S}=\tilde{S}_{1} \tilde{S}_{2}=\left(\bar{S}_{1}+\tilde{\theta}\right)\left(\bar{S}_{2}+\beta \tilde{\theta}\right)=\bar{S}_{1} \bar{S}_{2}+\left(\beta \bar{S}_{1}+\bar{S}_{2}\right) \tilde{\theta}+\beta \tilde{\theta}^{2}
$$

Given the assumed three-point probability distribution function of $\tilde{\theta}$, equation (11) can be written as

$$
\tilde{S}=\bar{S}_{1} \bar{S}_{2}+\left(\beta \bar{S}_{1}+\bar{S}_{2}+\beta \Theta\right) \tilde{\theta}+2 \beta \Theta \max (-\tilde{\theta}, 0) .
$$

Substituting equation (12) into equation (7) yields

$$
\begin{aligned}
\tilde{\Pi}= & \bar{S}_{1} \bar{S}_{2} X+P Z+\left[\left(\beta \bar{S}_{1}+\bar{S}_{2}+\beta \Theta\right) X-H\right] \tilde{\theta} \\
& +(2 \beta \Theta X-Z) \max (-\tilde{\theta}, 0) .
\end{aligned}
$$

Substituting $H=\left(\beta \bar{S}_{1}+\bar{S}_{2}+\beta \Theta\right) X$ and $Z=2 \beta \Theta X$ into equation (13) yields $\tilde{\Pi}=\bar{S}_{1} \bar{S}_{2} X+P Z$, which is non-stochastic. Thus, equations (8) and (9) hold simultaneously at $H^{*}=\left(\beta \bar{S}_{1}+\bar{S}_{2}+\beta \Theta\right) X$ and $Z^{*}=2 \beta \Theta X$ in this example.

Inspection of equation (12) reveals that the missing futures contract between the domestic and foreign currencies can be spanned by $\beta \bar{S}_{1}+\bar{S}_{2}+\beta \Theta$ of the currency futures contract and $2 \beta \Theta$ of the currency put options between the domestic

\footnotetext{
${ }^{11}$ Note that $\operatorname{Cov}(\tilde{\theta} \tilde{\epsilon}, \tilde{\theta})=\operatorname{Cov}[E(\tilde{\theta} \tilde{\epsilon} \mid \tilde{\theta}), \tilde{\theta}]=0$. Thus, $\left(\bar{S}_{1}+\tilde{\theta}\right) \tilde{\epsilon} X$ is indeed independent of $\tilde{\theta}$.
} 
and third currencies. Alternatively put, cross-hedging with the currency futures and put options completes the incomplete markets in the above example, thereby making risk sharing fully efficient.

In general, complete spanning of the missing futures contract is not feasible. To consider the hedging role of the currency put options, we first relax the condition of independent spot exchange rates, while retaining the condition of quadratic utility functions. To yield unambiguous results, we assume that $\tilde{\theta}$, thereby $\tilde{S}_{1}$, is symmetrically distributed. Let $G$ be the cumulative distribution function of $\tilde{\theta}$ over support $[-\Theta, \Theta]$, where $0<\Theta \leq \infty .{ }^{12}$ The following proposition shows the hedging role of currency options when the spot exchange rates, $\tilde{S}_{1}$ and $\tilde{S}_{2}$, are de facto dependent (i.e., $\beta \neq 0$ ).

Proposition 2. Suppose that the prices of the currency futures and put options between the domestic and third currencies are perceived as jointly unbiased by the multinational firm. If the firm has a quadratic utility function and if $\tilde{S}_{1}$ is symmetrically distributed, the firm's optimal cross-hedge position, $\left(H^{*}, Z^{*}\right)$, is given by

$$
\begin{aligned}
H^{*} & =\left(\beta \bar{S}_{1}+\bar{S}_{2}\right) X+\frac{Z^{*}}{2} \\
Z^{*} & =2 \beta X \frac{K-P \sigma^{2}}{\sigma^{2} / 2-2 P^{2}}
\end{aligned}
$$

where $\sigma^{2}=E\left(\tilde{\theta}^{2}\right), P=\int_{0}^{\Theta} \theta d G(\theta)$, and $K=\int_{0}^{\Theta} \theta^{3} d G(\theta)$. In particular, $4 \beta \Theta X<$ $Z^{*}<0$ when $\beta<0$ and $0<Z^{*}<4 \beta \Theta X$ when $\beta>0$.

The intuition of Proposition 2 is as follows. Under quadratic utility functions, the firm's optimal cross-hedge position, $\left(H^{*}, Z^{*}\right)$, minimizes the variability of its date 1 domestic currency income. Substituting equation (14) into equation (7) yields the firm's date 1 domestic currency income,

$$
\tilde{\Pi}^{*}=\bar{S}_{1} \bar{S}_{2} X+\left(\bar{S}_{1}+\tilde{\theta}\right) \tilde{\epsilon} X+(2 P-|\tilde{\theta}|) \frac{Z^{*}}{2}+\beta \tilde{\theta}^{2} X .
$$

Inspection of equation (16) reveals that the firm's optimal hedge position, $\left(H^{*}, Z^{*}\right)$, can be interpreted as selling $\left(\beta \bar{S}_{1}+\bar{S}_{2}\right) X$ currency futures and writing $Z^{*} / 2$ straddles at a price, $2 P$. Straddles are option strategies that combine calls and puts with the same strike prices and expiration. Since $\tilde{S}_{1}$ is symmetrically distributed, the unbiasedness of the futures price and put option premium implies that the synthesized straddles are also fairly priced. Note that the second term on the right-hand side of equation (16), $\left(\bar{S}_{1}+\tilde{\theta}\right) \tilde{\epsilon} X$, is independent of $\tilde{\theta}$ and thus is not hedgeable by trading currency futures and options. The last term on the right-hand side of equation (16), $\beta \tilde{\theta}^{2} X$, is however hedgeable. If $\beta<(>) 0$, this term would be concave (convex) and symmetric in $\tilde{\theta}$. This non-linear component of the firm's foreign exchange risk exposure can be closely matched by buying (writing) straddles, $Z^{*}<(>) 0$, which creates a convex (concave) and symmetric payoff to the

\footnotetext{
${ }^{12}$ The cumulative distribution function is symmetric if, and only if, $d G(\theta)=d G(-\theta)$ for all $\theta \in$ $[-\Theta, \Theta]$. For example, normal and uniform distribution functions are symmetric.
} 
firm's hedge position. Currency options play a hedging role because there is a quadratic source of uncertainty arising from the triangular parity condition when the spot exchange rates, $\tilde{S}_{1}$ and $\tilde{S}_{2}$, are de facto dependent. As such, linear payoff currency futures contracts no longer dominate non-linear payoff currency options for hedging purposes. For multinational firms with quadratic utility functions, Proposition 2 justifies the use of straddles as an optimal option strategy in the context of cross-hedging.

We now relax the condition of quadratic utility functions. As convincingly argued by Kimball (1990), (1993), prudence (i.e., $U^{\prime \prime \prime}>0$ ) is a reasonable behavioral assumption for decision making under multiple sources of uncertainty. It measures the propensity to prepare and forearm oneself under uncertainty, visà-vis risk aversion, which is how much one dislikes uncertainty and would turn away from it if one could. As shown by Leland (1968), Drèze and Modigliani (1972), and Kimball (1990), prudence is both necessary and sufficient to generate precautionary saving. Furthermore, prudence is implied by decreasing absolute risk aversion, which is instrumental in yielding many intuitively appealing comparative statics under uncertainty. Thus, it is of interest to see how the prudent firm would choose its optimal cross-hedge position, $\left(H^{*}, Z^{*}\right)$, in the following proposition.

Proposition 3. Suppose that the prices of the currency futures and put options between the domestic and third currencies are perceived as jointly unbiased by the multinational firm. If the firm is prudent and if $\tilde{S}_{1}$ is symmetrically distributed, the firm's optimal put option position, $Z^{*}$, is that $Z^{*}<0$ when $\beta \leq 0$ and $Z^{*}<$ $4 \beta \Theta X$ when $\beta>0$.

To see the intuition of Proposition 3, we recast equation (3) for the case where $\beta=0$,

$$
\tilde{\Pi}=\bar{S}_{1} \bar{S}_{2} X+\left(\bar{S}_{1}+\tilde{\theta}\right) \tilde{\epsilon} X+\tilde{\theta}\left(\bar{S}_{2} X-H\right)+[P-\max (-\tilde{\theta}, 0)] Z
$$

Given prudence (i.e., $U^{\prime \prime \prime}>0$ ), the firm is more sensitive to low realizations of its date 1 domestic currency income than to high ones (see Kimball (1990), (1993)). The firm as such has incentives to avoid the low realizations of its date 1 domestic currency income. Inspection of equation (17) reveals that the firm can achieve this goal by buying the currency put options, $Z^{*}<0$. Under the condition of independent spot exchange rates, the hedging role of currency options is driven entirely by the firm's non-linear marginal utility function. Due to this source of non-linearity when prudence prevails, non-linear payoff currency options have to be used to achieve better hedging performance. If both the conditions of quadratic utility functions and independent spot exchange rates are relaxed, two sources of non-linearity are present. When $\tilde{S}_{1}$ and $\tilde{S}_{2}$ are negatively correlated, each of these two sources of non-linearity calls for a long put option position, thereby making $Z^{*}<0$ optimal. When $\tilde{S}_{1}$ and $\tilde{S}_{2}$ are positively correlated, to reduce the variability of its date 1 domestic currency income, the firm has to write the currency put options. The hedging need due to prudence, however, induces the firm to buy the currency put options. As such, the optimal put option position is a priori ambiguous should $\tilde{S}_{1}$ and $\tilde{S}_{2}$ be positively correlated. 


\section{Optimal Dynamic Cross-Hedging}

In the previous section, we have focused exclusively on cross-hedging problems in a static setting. However, almost invariably, hedging problems faced by multinational firms are dynamic in nature. Typically, a stream of revenues or income flows needs to be hedged over a period of time with the flexibility that any hedge positions can be rebalanced from period to period. It is thus of practical importance to extend our analysis to solve a dynamic cross-hedging problem.

Consider a $T$-period extension of the static model developed in Section II. There is a stream of cash flows, $\left\{X_{1}, \ldots, X_{T}\right\}$, where $X_{t}$ is the net cash flow received by the firm from its foreign operation at the end of period $t(t=1, \ldots$, $T$ ). The spot exchange rate of the domestic currency against the foreign currency at the end of period $t$ is denoted by $\tilde{S}_{t}$. Let $\tilde{S}_{1, t}$ be the spot exchange rate of the domestic currency against the third currency at the end of period $t$. Likewise, let $\tilde{S}_{2, t}$ be the spot exchange rate of the third currency against the foreign currency at the end of period $t$. The law of one price implies that $\tilde{S}_{t}=\tilde{S}_{1, t} \tilde{S}_{2, t}$ for all $t=1$, $\ldots, T$.

For simplicity, we normalize the per-period riskless rate of interest to zero. ${ }^{13}$ We assume that $\tilde{S}_{1, t}$ is a martingale such that

$$
\tilde{S}_{1, t}=S_{1, t-1}+\tilde{\theta}_{t}
$$

where $S_{1, t-1}$ is the spot exchange rate realized at the end of period $t-1$, and $\tilde{\theta}_{t}$ is a zero mean, finite variance random variable independently distributed over time. Likewise, we assume that $\tilde{S}_{2, t}$ is a martingale such that

$$
\tilde{S}_{2, t}=S_{2, t-1}+\beta_{t}\left(\tilde{S}_{1, t}-S_{1, t-1}\right)+\tilde{\epsilon}_{t}=S_{2, t-1}+\beta_{t} \tilde{\theta}_{t}+\tilde{\epsilon}_{t}
$$

where $S_{2, t-1}$ is the spot exchange rate realized at the end of period $t-1, \beta_{t}$ is a constant, $\tilde{\epsilon}_{t}$ is a zero mean, finite variance random variable independently distributed over time and with respect to $\tilde{\theta}_{t}$, and the second equality follows from equation (18).

The firm's domestic currency income in period $t$ is given by

$$
\tilde{\Pi}_{t}=\tilde{S}_{1, t} \tilde{S}_{2, t} X_{t}+\left(F_{t}-\tilde{S}_{1, t}\right) H_{t}+\left[P_{t}-\max \left(S_{1, t-1}-\tilde{S}_{1, t}, 0\right)\right] Z_{t}
$$

where $H_{t}$ is the number of the currency futures sold (purchased if negative) at the futures price, $F_{t}$, in period $t$; and $Z_{t}$ is the number of the currency put options sold (purchased if negative) with the strike price, $S_{1, t-1}$, at the option premium, $P_{t}$, in period $t$. As in the previous section, to focus on the hedging role of the currency futures and put options, we assume that the futures price and put option premium in each period are perceived as unbiased by the firm. That is, $F_{t}=S_{1, t-1}$ and $P_{t}=E\left[\max \left(-\tilde{\theta}_{t}, 0\right)\right]$ for all $t=1, \ldots, T$.

\footnotetext{
${ }^{13}$ As long as the process of riskless rates of interest is non-stochastic, this normalization is without any loss of generality.
} 
The firm's dynamic decision problem is to choose its cross-hedge position in each period, conditional on all available information in that period and before, so as to maximize the expected utility of its terminal wealth, $\tilde{W}_{T},{ }^{14}$

$$
\max _{\left\{H_{t}, Z_{t}\right\}} E\left[U\left(\tilde{W}_{T}\right)\right]
$$

Since the per-period riskless rate of interest is normalized to zero, the firm's terminal wealth is simply equal to the sum of all domestic currency income from period 1 to period $T$, i.e., $\tilde{W}_{T}=\sum_{\tau=1}^{T} \tilde{\Pi}_{\tau}$.

To solve the firm's dynamic decision problem, program (21), we use backward induction. Specifically, in the final period, the firm's maximum expected utility of its terminal wealth given its information set in period $T, \Omega_{T}$, is denoted by

$$
V_{T}\left(W_{T-1} \mid \Omega_{T}\right)=\max _{H_{T}, Z_{T}} E\left[U\left(W_{T-1}+\tilde{\Pi}_{T}\right) \mid \Omega_{T}\right]
$$

where $W_{T-1}=\sum_{\tau=1}^{T-1} \Pi_{\tau}$ is the total realized domestic currency income from period 1 to period $T-1, E\left(\cdot \mid \Omega_{T}\right)$ is the expectation operator conditional on $\Omega_{T}$, and $\tilde{\Pi}_{T}$ is defined in equation (20) for $t=T$. In period $T-1$, the firm's maximum expected utility of its terminal wealth given its information set in period $T-1$, $\Omega_{T-1}$, is denoted by

$$
V_{T-1}\left(W_{T-2} \mid \Omega_{T-1}\right)=\max _{H_{T-1}, Z_{T-1}} E\left[V_{T}\left(W_{T-2}+\tilde{\Pi}_{T-1} \mid \Omega_{T}\right) \mid \Omega_{T-1}\right]
$$

where $V_{T}$ is defined in equation (22), $W_{T-2}=\sum_{\tau=1}^{T-2} \Pi_{\tau}$ is the total realized domestic currency income from period 1 to period $T-2, E\left(\cdot \mid \Omega_{T-1}\right)$ is the expectation operator conditional on $\Omega_{T-1}$, and $\tilde{\Pi}_{T-1}$ is defined in equation (20) for $t=T-1$. In general, in any period $t$, the firm's maximum expected utility of its terminal wealth given its information set in period $t, \Omega_{t}$, is denoted by

$$
V_{t}\left(W_{t-1} \mid \Omega_{t}\right)=\max _{H_{t}, Z_{t}} E\left[V_{t+1}\left(W_{t-1}+\tilde{\Pi}_{t} \mid \Omega_{t+1}\right) \mid \Omega_{t}\right],
$$

where $W_{t-1}=\sum_{\tau=1}^{t-1} \Pi_{\tau}$ is the total realized domestic currency income from period 1 to period $t-1$ with $W_{0}=0, E\left(\cdot \mid \Omega_{t}\right)$ is the expectation operator conditional on $\Omega_{t}$, and $\tilde{\Pi}_{t}$ is defined in equation (20).

To yield an analytical solution to the dynamic cross-hedging problem as specified in equations (22) and (24), we shall focus on the case where the firm's utility function is quadratic and $\tilde{\theta}_{t}$, thereby $\tilde{S}_{1, t}$, is symmetrically distributed for all $t=1, \ldots, T$. Let $G_{t}$ be the cumulative distribution function of $\tilde{\theta}_{t}$ over support $\left[-\Theta_{t}, \Theta_{t}\right]$, where $0<\Theta_{t} \leq \infty$. The following proposition shows that the dynamic solution is analogous to the static solution as reported in Proposition 2.

Proposition 4. Suppose that the prices of the currency futures and put options between the domestic and third currencies are perceived as jointly unbiased by the multinational firm in each period. If the firm has a quadratic utility function

\footnotetext{
${ }^{14}$ This is the simplest formulation of the standard dynamic asset allocation problem. See Duffie and Richardson (1991) and Haugh and Lo (2001).
} 
and if $\tilde{S}_{1, t}$ is symmetrically distributed, the firm's optimal cross-hedge position, $\left(H_{t}^{*}, Z_{t}^{*}\right)$, in period $t$ is given by

$$
\begin{aligned}
H_{t}^{*} & =\left(\beta_{t} S_{1, t-1}+S_{2, t-1}\right) \sum_{\tau=t}^{T} X_{\tau}+\frac{Z_{t}^{*}}{2}, \\
Z_{t}^{*} & =2 \beta_{t} \sum_{\tau=t}^{T} X_{\tau} \frac{K_{t}-P_{t} \sigma_{t}^{2}}{\sigma_{t}^{2} / 2-2 P_{t}^{2}},
\end{aligned}
$$

where $\sigma_{t}^{2}=E\left(\tilde{\theta}_{t}^{2}\right), P_{t}=\int_{0}^{\Theta_{t}} \theta_{t} d G_{t}\left(\theta_{t}\right)$, and $K_{t}=\int_{0}^{\Theta_{t}} \theta_{t}^{3} d G_{t}\left(\theta_{t}\right)$. In particular, $4 \beta_{t} \Theta_{t} \sum_{\tau=t}^{T} X_{\tau}<Z_{t}^{*}<0$ when $\beta_{t}<0$ and $0<Z_{t}^{*}<4 \beta_{t} \Theta_{t} \sum_{\tau=t}^{T} X_{\tau}$ when $\beta_{t}>0$.

The intuition of Proposition 4 is as follows. In the final period (i.e., period $T$ ), the firm's cross-hedging decision problem is a static one and thus the results of Proposition 2 apply. In period $T-1$, the firm anticipates that

$$
\tilde{\Pi}_{T}^{*}=\tilde{S}_{1, T-1} \tilde{S}_{2, T-1} X_{T}+\left(\tilde{S}_{1, T-1}+\tilde{\theta}_{T}\right) \tilde{\epsilon}_{T} X_{T}+\left(2 P_{T}-\left|\tilde{\theta}_{T}\right|\right) \frac{Z_{T}^{*}}{2}+\beta_{T} \tilde{\theta}_{T}^{2} X_{T}
$$

where we have substituted equations (25) and (26) into equation (20) for $t=T$. The firm's terminal wealth is thus $W_{T-2}+\tilde{\Pi}_{T-1}+\tilde{\Pi}_{T}^{*}$, which is equal to

$$
\begin{aligned}
W_{T-2} & +\tilde{S}_{1, T-1} \tilde{S}_{2, T-1}\left(X_{T-1}+X_{T}\right)-\tilde{\theta}_{T-1} H_{T-1} \\
& +\left[P_{T-1}-\max \left(-\tilde{\theta}_{T-1}, 0\right)\right] Z_{T-1} \\
& +\left(\tilde{S}_{1, T-2}+\tilde{\theta}_{T-1}+\tilde{\theta}_{T}\right) \tilde{\epsilon}_{T} X_{T}+\left(2 P_{T}-\left|\tilde{\theta}_{T}\right|\right) \frac{Z_{T}^{*}}{2}+\beta_{T} \tilde{\theta}_{T}^{2} X_{T} .
\end{aligned}
$$

Given that the firm has a quadratic utility function, its optimal cross-hedge position, $\left(H_{T-1}^{*}, Z_{T-1}^{*}\right)$, minimizes the variability of its terminal wealth. Since the last three terms of the above expression are independent of $\tilde{\theta}_{T-1}$, they are not hedgeable by trading currency futures and options in period $T-1$. Thus, the firm's cross-hedging decision problem reduces to a static one by replacing $X_{T-1}$ with $X_{T-1}+X_{T}$. Similar logic applies to other periods, rendering a close analogy between the dynamic and static solutions.

Two remarks are in order. First, as evident from equations (25) and (26), the optimal dynamic cross-hedge position in period $t$ is computed from the joint probability distribution function of $\tilde{S}_{1, t}$ and $\tilde{S}_{2, t}$. In other words, even in the case where $X_{1}=\ldots=X_{T-1}=0$ and $X_{T}=X$, the optimal dynamic cross-hedge position would be time varying as long as the time series of $\tilde{S}_{1}$ and $\tilde{S}_{2}$ are non-stationary. Second, inspection of equations (25) and (26) reveals that the optimal dynamic cross-hedge position in period $t$ depends not only on the net cash flow in that period, $X_{t}$, but also on the net cash flows in all subsequent periods, $X_{t+1}, \ldots$, $X_{T}$. Thus, even under stationary time series of $\tilde{S}_{1}$ and $\tilde{S}_{2}$, the optimal dynamic cross-hedge position remains time varying. In this regard, the dynamic solution is rather different from the static solution to the cross-hedging problem. 


\section{Hedge Effectiveness}

In this section, we shall evaluate the potential usefulness of cross-hedging with currency futures and options. Suppose that the firm has a quadratic utility function. Given the joint unbiasedness of the prices of the currency futures and put options, we know that the firm's expected utility is a linear function of the variance of the date 1 domestic currency income of the firm. In other words, the optimal cross-hedge position as reported in Proposition 2 minimizes the variance of the firm's date 1 domestic currency income. The hedge effectiveness of the optimal cross-hedge position as such can be gauged by the difference between the variances with and without cross-hedging.

Assume that $\tilde{\theta}$ is normally distributed with mean zero and variance $\sigma^{2}$. Then, we have

$$
\begin{aligned}
& P=\int_{0}^{\infty} \theta \frac{e^{-\theta^{2} / 2 \sigma^{2}}}{\sqrt{2 \pi} \sigma} d \theta=\frac{\sigma}{\sqrt{2 \pi}}, \quad \text { and } \\
& K=\int_{0}^{\infty} \theta^{3} \frac{e^{-\theta^{2} / 2 \sigma^{2}}}{\sqrt{2 \pi} \sigma} d \theta=\frac{\sqrt{2} \sigma^{3}}{\sqrt{\pi}} .
\end{aligned}
$$

Using this fact and Proposition 2, we have

$$
H^{*}=\left(\beta \bar{S}_{1}+\bar{S}_{2}\right) X+\frac{Z^{*}}{2}, \text { and } Z^{*}=2 \beta X \sigma \frac{\sqrt{2 \pi}}{\pi-2} .
$$

It is easily verified that in the absence of the currency put options (i.e., $Z \equiv 0$ ), the optimal futures position that minimizes the variance of the firm's date 1 domestic currency income is given by ${ }^{15}$

$$
H^{0}=\left(\beta \bar{S}_{1}+\bar{S}_{2}\right) X
$$

The empirical exercise is as follows. We refer to Japan as the home country, Taiwan as the foreign country, and the U.S. as the third country. The foreign currency cash flow, $X$, is arbitrarily set equal to 100 Taiwan dollars. The data on daily spot exchange rates are supplied by Datastream. Daily spot exchange rates for the Japanese yen $\left(\tilde{S}_{1}\right)$ and the Taiwan dollar $\left(1 / \tilde{S}_{2}\right)$, both against the U.S. dollar, are taken over the period beginning on January 1, 1997, and ending on April 10, 2001. Based on equation (2), we regress, each year, the de-meaned U.S. dollar/Taiwan dollar series on the de-meaned Japanese yen/U.S. dollar series to obtain five estimates of $\beta$ over the sample period. All regression estimates of $\beta$ are negative and significantly different from zero at the $1 \%$ level. In each year, we substitute the sample means of the two exchange rates and the regression estimate of $\beta$ into equations (27) and (28) to compute the firm's optimal cross-hedge positions, $\left(H^{*}, Z^{*}\right)$ and $H^{0}$, respectively. These results are reported in Table 1.

To compare the performances of using currency futures and options for crosshedging purposes, we need to estimate the variances of the firm's date 1 domestic currency income,

$$
\operatorname{Var}\left\{\tilde{S}_{1} \tilde{S}_{2} X+\left(F-\tilde{S}_{1}\right) H+\left[P-\max \left(\bar{S}_{1}-\tilde{S}_{1}, 0\right)\right] Z\right\},
$$

\footnotetext{
${ }^{15}$ The optimal futures position is easily derived by using the proof of Proposition 2 with $Z \equiv 0$.
} 
TABLE 1

Data Description

\begin{tabular}{|c|c|c|c|c|c|}
\hline & 1997 & 1998 & 1999 & 2000 & 2001 \\
\hline Observations & 261 & 261 & 261 & 260 & 72 \\
\hline \multicolumn{6}{|c|}{ Japanese Yen/U.S. Dollar } \\
\hline Mean & 121.03 & 130.78 & 113.67 & 107.81 & 118.83 \\
\hline Standard Deviation & 4.74 & 8.87 & 6.97 & 2.10 & 3.40 \\
\hline \multicolumn{6}{|c|}{ Taiwan Dollar/U.S. Dollar } \\
\hline Mean & 28.71 & 33.46 & 32.26 & 31.23 & 32.56 \\
\hline Standard Deviation & 1.63 & 0.91 & 0.50 & 0.78 & 0.26 \\
\hline Estimates of $\beta$ & $-21.61^{*}$ & $-7.53^{*}$ & $-5.96^{*}$ & $-24.81^{*}$ & $-2.87^{*}$ \\
\hline \multicolumn{6}{|c|}{ Optimal Cross-Hedge Positions } \\
\hline$H^{0}$ & 0.88 & 2.01 & 2.42 & 0.53 & 2.73 \\
\hline$H^{*}$ & 0.65 & 1.86 & 2.33 & 0.42 & 2.71 \\
\hline$Z^{*}$ & -0.45 & -0.29 & -0.18 & -0.23 & -0.04 \\
\hline
\end{tabular}

Table 1 presents descriptive statistics on the two spot exchange rate series from January 1, 1997, to April 10, 2001. Regression estimates of $\beta$ are in the unit of $10^{-5}$ and are marked ${ }^{*}$ to indicate significance at the $1 \%$ level. Optimal cross-hedge positions are computed from equations (27) and (28) in the text.

for three cases: i) no hedging (i.e., $H=Z=0$ ), ii) hedging with futures only (i.e., $H=H^{0}$ and $Z=0$ ), and iii) hedging with futures and options (i.e., $H=H^{*}$ and $Z=Z^{*}$ ). To this end, we compute, each year, three sample variances of the firm's date 1 domestic currency income using the actual realized exchange rates during the year. The first sample variance is based on $H=Z=0$. The second sample variance is based on $H=H^{0}$ and $Z=0$, where $H^{0}$ is taken from Table 1 . The third sample variance is based on $H=H^{*}$ and $Z=Z^{*}$, where $\left(H^{*}, Z^{*}\right)$ is taken from Table 1. These results are reported in Table 2.

TABLE 2

Comparisons of Hedge Effectiveness

\begin{tabular}{|c|c|c|c|c|c|}
\hline & 1997 & 1998 & 1999 & 2000 & 2001 \\
\hline \multicolumn{6}{|l|}{ Portfolio Variances } \\
\hline No Hedging & 387.41 & 328.63 & 282.49 & 42.54 & 83.50 \\
\hline Hedging with Futures Only & 380.17 & 41.92 & 15.63 & 41.82 & 10.05 \\
\hline Hedging with Futures and Options & 372.04 & 39.68 & 15.46 & 41.31 & 9.99 \\
\hline \multicolumn{6}{|c|}{ Variance Reduction of Hedging with Futures and Options Compared to: } \\
\hline No Hedging & $3.97 \%$ & $87.93 \%$ & $94.53 \%$ & $2.89 \%$ & $88.04 \%$ \\
\hline Hedging with Futures Only & $2.14 \%$ & $5.34 \%$ & $1.09 \%$ & $1.22 \%$ & $0.60 \%$ \\
\hline
\end{tabular}

As Table 2 shows, using currency futures and options for cross-hedging purposes on average results in variance reductions of about $56 \%$ compared to the no hedging strategy and about $2 \%$ compared to using currency futures as the sole hedging instrument. Indeed, it is evident from Table 2 that currency options always offer firms an improved ability to manage their foreign exchange risk exposure compared to using currency futures only. The improvements in hedge effectiveness can be substantial (more than 5\%). 


\section{Conclusions}

In the post-Bretton Woods era, exchange rates have been increasingly volatile, making foreign exchange risk management a fact of financial life. This paper studies how a risk-averse multinational firm, confronting a foreign currency cash flow but possessing no direct hedging opportunities, can employ derivative securities on related currencies to reduce its foreign exchange risk exposure. Currency options play no role as a hedging instrument only under restrictive conditions of quadratic utility functions and independent spot exchange rates. If either the spot exchange rates are correlated or the firm is prudent in the sense of Kimball (1990), (1993), we have shown that the firm would optimally use currency options for hedging purposes. We thus add to the scant literature on the hedging role of options in that currency options are used by multinational firms in the case of currency mismatching.

Cross-hedging is important because it expands the opportunity set of hedging alternatives. Given the fact that currency derivative markets are not readily available in less developed countries and are just starting to develop in many of the newly industrializing countries of Latin America and Asia Pacific, it is clear that, for many multinational firms exposed to currencies of these countries, crosshedging will continue to be a major risk management technique for the reduction of their foreign exchange risk exposure.

\section{Appendix}

\section{Proof of Proposition 1}

If $\beta=0$, substituting $H^{*}=\bar{S}_{2} X$ and $Z^{*}=0$ into equation (3) yields $\tilde{\Pi}^{*}=$ $\bar{S}_{1} \bar{S}_{2} X+\left(\bar{S}_{1}+\tilde{\theta}\right) \tilde{\epsilon} X$. Let $U(\Pi)=\Pi-b \Pi^{2}$, where $b$ is a positive constant. Partially differentiating $E\left[U^{\prime}\left(\tilde{\Pi}^{*}\right) \mid \theta\right]$ with respect to $\theta$ yields

$$
\frac{\partial E\left[U^{\prime}\left(\tilde{\Pi}^{*}\right) \mid \theta\right]}{\partial \theta}=E(-2 b \tilde{\epsilon} X \mid \theta)=0
$$

since $\tilde{\epsilon}$ has zero mean and is independent of $\tilde{\theta}$. Thus, $E\left[U^{\prime}\left(\tilde{\Pi}^{*}\right) \mid \theta\right]$ is invariant to $\theta$ when $H^{*}=\bar{S}_{2} X$ and $Z^{*}=0$. In other words, equations (8) and (9) hold simultaneously when $H^{*}=\bar{S}_{2} X$ and $Z^{*}=0$.

Proof of Proposition 2

Let $U(\Pi)=\Pi-b \Pi^{2}$, where $b$ is a positive constant. Then, equation (8) becomes

$$
\operatorname{Cov}\left[E\left(1-2 b \tilde{\Pi}^{*} \mid \tilde{\theta}\right), \tilde{\theta}\right]=0
$$

Since $\tilde{\theta}$ and $\tilde{\epsilon}$ have zero mean and are independent of each other, the above equation can be written as

$$
\int_{-\Theta}^{0} \Pi_{1}^{*}(\theta) \theta d G(\theta)+\int_{0}^{\Theta} \Pi_{2}^{*}(\theta) \theta d G(\theta)=0
$$


where $\Pi_{1}^{*}(\theta)=\Pi_{2}^{*}(\theta)+\theta Z^{*}$ and $\Pi_{2}^{*}(\theta)=\left(\bar{S}_{1}+\theta\right)\left(\bar{S}_{2}+\beta \theta\right) X-\theta H^{*}+P Z^{*}$. Since $\tilde{\theta}$ is symmetrically distributed, we have $d G(\theta)=d G(-\theta)$ for all $\theta \in[-\Theta, \Theta]$. Using this fact, we can write equation (A-1) as

$$
\int_{0}^{\Theta}\left[\Pi_{2}^{*}(\theta)-\Pi_{1}^{*}(-\theta)\right] \theta d G(\theta)=0 .
$$

Note that

$$
\Pi_{2}^{*}(\theta)-\Pi_{1}^{*}(-\theta)=2 \theta\left[\left(\beta \bar{S}_{1}+\bar{S}_{2}\right) X-H^{*}+\frac{Z^{*}}{2}\right] .
$$

Substituting equation (A-3) into equation (A-2) yields

$$
2\left[\left(\beta \bar{S}_{1}+\bar{S}_{2}\right) X-H^{*}+\frac{Z^{*}}{2}\right] \int_{0}^{\Theta} \theta^{2} d G(\theta)=0,
$$

which implies that $H^{*}$ is given by equation (14) in Proposition 2.

Since $U$ is quadratic and $\tilde{\theta}$ is symmetrically distributed, using a similar procedure as above, we can write equation (9) as

$$
\int_{0}^{\Theta} \Pi_{2}^{*}(\theta) P d G(\theta)+\int_{0}^{\Theta} \Pi_{1}^{*}(-\theta)(P-\theta) d G(\theta)=0 .
$$

Subtracting equation (A-2) from two times equation (A-4) yields

$$
\int_{0}^{\Theta}\left[\Pi_{2}^{*}(\theta)+\Pi_{1}^{*}(-\theta)\right](2 P-\theta) d G(\theta)=0 .
$$

Using equation (14), we have

$$
\Pi_{2}^{*}(\theta)+\Pi_{1}^{*}(-\theta)=2 \bar{S}_{1} \bar{S}_{2} X+2 \beta \theta^{2} X+2 P Z^{*}-\theta Z^{*} .
$$

Since $P=E[\max (-\tilde{\theta}, 0)]$, the symmetric distribution of $\tilde{\theta}$ implies that $\int_{0}^{\Theta}(2 P-$ $\theta) d G(\theta)=0$. Using this fact and substituting equation (A-6) into equation (A-5) yields

$$
\int_{0}^{\Theta}\left(2 \beta \theta^{2} X-\theta Z^{*}\right)(2 P-\theta) d G(\theta)=0,
$$

which implies that $Z^{*}$ is given by equation (15) in Proposition 2 . Since $P=$ $\int_{0}^{\Theta} \theta d G(\theta)<\Theta / 2,2 P+\theta<2 \Theta$ for all $\theta \in[0, \Theta]$. It then follows from equation (15) that $Z^{*} \in(4 \beta \Theta X, 0)$ if $\beta<0$ and $Z^{*} \in(0,4 \beta \Theta X)$ if $\beta>0$.

\section{Proof of Proposition 3}

Since $\tilde{\theta}$ is symmetrically distributed, we have $d G(\theta)=d G(-\theta)$ for all $\theta \in$ $[-\Theta, \Theta]$. Using this fact, we can write equations (8) and (9) as

$$
\int_{0}^{\Theta} E\left\{U^{\prime}\left[\tilde{\Pi}_{2}^{*}(\theta)\right]-U^{\prime}\left[\tilde{\Pi}_{1}^{*}(-\theta)\right]\right\} \theta d G(\theta)=0
$$

$$
\int_{0}^{\Theta}\left\{E\left\{U^{\prime}\left[\tilde{\Pi}_{2}^{*}(\theta)\right]\right\} P+E\left\{U^{\prime}\left[\tilde{\Pi}_{1}^{*}(-\theta)\right]\right\}(P-\theta)\right\} d G(\theta)=0
$$


where $\tilde{\Pi}_{1}^{*}(\theta)=\tilde{\Pi}_{2}^{*}(\theta)+\theta Z^{*}$ and $\tilde{\Pi}_{2}^{*}(\theta)=\left(\bar{S}_{1}+\theta\right)\left(\bar{S}_{2}+\beta \theta+\tilde{\epsilon}\right) X-\theta H^{*}+P Z^{*}$. Subtracting equation (A-7) from two times equation (A-8) yields

$$
\int_{0}^{\Theta} E\left\{U^{\prime}\left[\tilde{\Pi}_{2}^{*}(\theta)\right]+U^{\prime}\left[\tilde{\Pi}_{1}^{*}(-\theta)\right]\right\}(2 P-\theta) d G(\theta)=0 .
$$

Define $\Gamma(\theta)=E\left\{U^{\prime}\left[\tilde{\Pi}_{2}^{*}(\theta)\right]+U^{\prime}\left[\tilde{\Pi}_{1}^{*}(-\theta)\right]\right\}$. Since $P=E[\max (-\tilde{\theta}, 0)]$, the symmetric distribution of $\tilde{\theta}$ implies that $\int_{0}^{\Theta}(2 P-\theta) d G(\theta)=0$. Using this fact and the definition of $\Gamma(\theta)$, equation (A-9) can be written as

$$
\int_{0}^{\Theta}[\Gamma(\theta)-\Gamma(2 P)](2 P-\theta) d G(\theta)=0 .
$$

Differentiating $\Gamma(\theta)$ with respect to $\theta$ yields

$$
\begin{aligned}
\Gamma^{\prime}(\theta)= & E\left\{U^{\prime \prime}\left[\tilde{\Pi}_{2}^{*}(\theta)\right]\left[\left(\beta \bar{S}_{1}+\bar{S}_{2}+2 \beta \theta+\tilde{\epsilon}\right) X-H^{*}\right]\right. \\
& \left.-U^{\prime \prime}\left[\tilde{\Pi}_{1}^{*}(-\theta)\right]\left[\left(\beta \bar{S}_{1}+\bar{S}_{2}-2 \beta \theta+\tilde{\epsilon}\right) X-H^{*}+Z^{*}\right]\right\} \\
= & E\left\{\left\{U^{\prime \prime}\left[\tilde{\Pi}_{2}^{*}(\theta)\right]-U^{\prime \prime}\left[\tilde{\Pi}_{1}^{*}(-\theta)\right]\right\}\right. \\
& \left.\times\left[\left(\beta \bar{S}_{1}+\bar{S}_{2}+\tilde{\epsilon}\right) X-H^{*}+\frac{Z^{*}}{2}\right]\right\} \\
& +\frac{1}{2} E\left\{U^{\prime \prime}\left[\tilde{\Pi}_{2}^{*}(\theta)\right]+U^{\prime \prime}\left[\tilde{\Pi}_{1}^{*}(-\theta)\right]\right\}\left(4 \beta \theta X-Z^{*}\right) .
\end{aligned}
$$

Note that

$$
\tilde{\Pi}_{2}^{*}(\theta)-\tilde{\Pi}_{1}^{*}(-\theta)=2 \theta\left[\left(\beta \bar{S}_{1}+\bar{S}_{2}+\tilde{\epsilon}\right) X-H^{*}+\frac{Z^{*}}{2}\right] .
$$

Using this fact and prudence (i.e., $U^{\prime \prime \prime}>0$ ), the first term on the right-hand side of equation (A-11) is positive. If $\beta \leq 0$, it follows from equation (A-11) that $\Gamma^{\prime}(\theta)>0$ for all $\theta \in[0, \Theta]$ if $Z^{*} \geq 0$. Thus, $\Gamma(\theta)-\Gamma(2 P)>(<) 0$ whenever $2 P-\theta<(>) 0$, implying that the left-hand side of equation (A-10) is negative, which is a contradiction. In other words, for equation (A-10) to hold, it must be true that $Z^{*}<0$ when $\beta \leq 0$. If $\beta>0$, equation (A-11) implies that $\Gamma^{\prime}(\theta)>0$ for all $\theta \in[0, \Theta]$ if $Z^{*} \geq 4 \beta \Theta X$. Thus, $\Gamma(\theta)-\Gamma(2 P)>(<) 0$ whenever $2 P-\theta<(>) 0$, implying that the left-hand side of equation (A-10) is negative, which is a contradiction. In other words, for equation (A-10) to hold when $\beta>0$, it must be true that $Z^{*}<4 \beta \Theta X$.

\section{Proof of Proposition 4}

Let $U\left(W_{T}\right)=W_{T}-b W_{T}^{2}$, where $b$ is a positive constant. We shall prove this proposition by the principle of mathematical induction.

We start with the final period. Given the martingale assumption, $\Omega_{T}$ is as informative as the pair of the realized spot exchange rates at the end of period $T$ $1,\left(S_{1, T-1}, S_{2, T-1}\right)$. Thus, the first-order conditions for the optimization problem on the right-hand side of equation (22) are given by

$$
\begin{aligned}
E\left\{\left[1-2 b\left(W_{T-1}+\tilde{\Pi}_{T}^{*}\right)\right] \tilde{\theta}_{T} \mid S_{1, T-1}, S_{2, T-1}\right\} & =0, \\
E\left\{\left[1-2 b\left(W_{T-1}+\tilde{\Pi}_{T}^{*}\right)\right]\left[P_{T}-\max \left(-\tilde{\theta}_{T}, 0\right)\right] \mid S_{1, T-1}, S_{2, T-1}\right\} & =0 .
\end{aligned}
$$


Given that $E\left(\tilde{\theta}_{T}\right)=E\left(\tilde{\epsilon}_{T}\right)=0, P_{T}=E\left[\max \left(-\tilde{\theta}_{T}, 0\right)\right]$, and $\tilde{\theta}_{T}$ and $\tilde{\epsilon}_{T}$ are independent, the above two equations reduce to

$$
\begin{array}{r}
\int_{-\Theta_{T}}^{0} \Pi_{1 T}^{*}\left(\theta_{T}\right) \theta_{T} d G_{T}\left(\theta_{T}\right)+\int_{0}^{\Theta_{T}} \Pi_{2 T}^{*}\left(\theta_{T}\right) \theta_{T} d G_{T}\left(\theta_{T}\right)=0 \\
\int_{-\Theta_{T}}^{0} \Pi_{1 T}^{*}\left(\theta_{T}\right)\left(P_{T}+\theta_{T}\right) d G_{T}\left(\theta_{T}\right)+\int_{0}^{\Theta_{T}} \Pi_{2 T}^{*}\left(\theta_{T}\right) P_{T} d G_{T}\left(\theta_{T}\right)=0
\end{array}
$$

where $\Pi_{1 T}^{*}\left(\theta_{T}\right)=\Pi_{2 T}^{*}\left(\theta_{T}\right)+\theta_{T} Z_{T}^{*}$ and $\Pi_{2 T}^{*}\left(\theta_{T}\right)=\left(S_{1, T-1}+\theta_{T}\right)\left(S_{2, T-1}+\beta_{T} \theta_{T}\right) X_{T}-$ $\theta_{T} H_{T}^{*}+P_{T} Z_{T}^{*}$. It then follows from the proof of Proposition 2 that equations (25) and (26) for $t=T$ are indeed the solution to the optimization problem on the right-hand side of equation (22). The firm's optimal domestic currency income in period $T$, conditional on $\left(S_{1, T-1}, S_{2, T-1}\right)$, is given by

$$
\begin{aligned}
\tilde{\Pi}_{T}^{*}= & S_{1, T-1} S_{2, T-1} X_{T}+\left(S_{1, T-1}+\tilde{\theta}_{T}\right) \tilde{\epsilon}_{T} X_{T} \\
& +\left(2 P_{T}-\left|\tilde{\theta}_{T}\right|\right) \frac{Z_{T}^{*}}{2}+\beta_{T} \tilde{\theta}_{T}^{2} X_{T},
\end{aligned}
$$

where we have substituted equations (25) and (26) into equation (20) for $t=T$. Thus, we have

$$
\begin{aligned}
& V_{T}\left(W_{T-1} \mid S_{1, T-1}, S_{2, T-1}\right)= \\
& \quad E\left[U\left(W_{T-1}+\tilde{\Pi}_{T}^{*}\right) \mid S_{1, T-1}, S_{2, T-1}\right],
\end{aligned}
$$

where $\tilde{\Pi}_{T}^{*}$ is defined in equation (A-12).

In period $T-1$, it follows from the martingale assumption that $\Omega_{T-1}$ is as informative as $\left(S_{1, T-2}, S_{2, T-2}\right)$. Using equation (A-13), the firm's decision problem as described on the right-hand side of equation (23) becomes

$$
\begin{gathered}
\max _{H_{T-1}, Z_{T-1}} E\left\{E\left[U\left(W_{T-2}+\tilde{\Pi}_{T-1}+\tilde{\Pi}_{T}^{*}\right) \mid \tilde{S}_{1, T-1}, \tilde{S}_{2, T-1}\right]\right. \\
\left.\mid S_{1, T-2}, S_{2, T-2}\right\} .
\end{gathered}
$$

Conditional on $\left(S_{1, T-2}, S_{2, T-2}\right)$, it follows from equation (20) for $t=T-1$ and equation (A-12) that

$$
\begin{aligned}
\tilde{\Pi}_{T-1}+\tilde{\Pi}_{T}^{*}= & \left(S_{1, T-2}+\tilde{\theta}_{T-1}\right)\left(S_{2, T-2}+\beta_{T-1} \tilde{\theta}_{T-1}+\tilde{\epsilon}_{T-1}\right)\left(X_{T-1}+X_{T}\right) \\
& -\tilde{\theta}_{T-1} H_{T-1}+\left[P_{T-1}-\max \left(-\tilde{\theta}_{T-1}, 0\right)\right] Z_{T-1} \\
& +\left(S_{1, T-2}+\tilde{\theta}_{T-1}+\tilde{\theta}_{T}\right) \tilde{\epsilon}_{T} X_{T}+\left(2 P_{T}-\left|\tilde{\theta}_{T}\right|\right) \frac{Z_{T}^{*}}{2}+\beta_{T} \tilde{\theta}_{T}^{2} X_{T}
\end{aligned}
$$

The first-order conditions for program (A-14) are given by

$$
\begin{aligned}
E\left\{\left[1-2 b\left(W_{T-2}+\tilde{\Pi}_{T-1}^{*}+\tilde{\Pi}_{T}^{*}\right)\right] \tilde{\theta}_{T-1} \mid S_{1, T-2}, S_{2, T-2}\right\} & =0, \\
E\left\{\left[1-2 b\left(W_{T-2}+\tilde{\Pi}_{T-1}^{*}+\tilde{\Pi}_{T}^{*}\right)\right]\left[P_{T-1}-\max \left(-\tilde{\theta}_{T-1}, 0\right)\right] \mid S_{1, T-2}, S_{2, T-2}\right\} & =0 .
\end{aligned}
$$


Note first that $E\left(\tilde{\theta}_{T-1}\right)=E\left(\tilde{\theta}_{T}\right)=E\left(\tilde{\epsilon}_{T-1}\right)=E\left(\tilde{\epsilon}_{T}\right)=0, P_{T-1}=E\left[\max \left(-\tilde{\theta}_{T-1}, 0\right)\right]$, and $\tilde{\theta}_{T-1}$ is independent of $\tilde{\theta}_{T}, \tilde{\epsilon}_{T-1}$, and $\tilde{\epsilon}_{T}$. Note also that $P_{T}$ and $Z_{T}^{*}$ are invariant to different realizations of $\tilde{\theta}_{T-1}$. Thus, the above two equations reduce to

$$
\begin{gathered}
\int_{-\Theta_{T-1}}^{0} \Pi_{1 T-1}^{*}\left(\theta_{T-1}\right) \theta_{T-1} d G_{T-1}\left(\theta_{T-1}\right) \\
+\int_{0}^{\Theta_{T-1}} \Pi_{2 T-1}^{*}\left(\theta_{T-1}\right) \theta_{T-1} d G_{T-1}\left(\theta_{T-1}\right)=0 \\
\int_{-\Theta_{T-1}}^{0} \Pi_{1 T-1}^{*}\left(\theta_{T-1}\right)\left(P_{T-1}+\theta_{T-1}\right) d G_{T-1}\left(\theta_{T-1}\right) \\
+\int_{0}^{\Theta_{T-1}} \Pi_{2 T-1}^{*}\left(\theta_{T-1}\right) P_{T-1} d G_{T-1}\left(\theta_{T-1}\right)=0
\end{gathered}
$$

where $\Pi_{1 T-1}^{*}\left(\theta_{T-1}\right)=\Pi_{2 T-1}^{*}\left(\theta_{T-1}\right)+\theta_{T-1} Z_{T-1}^{*}$ and $\Pi_{2 T-1}^{*}\left(\theta_{T-1}\right)=\left(S_{1, T-2}+\right.$ $\left.\theta_{T-1}\right)\left(S_{2, T-2}+\beta_{T-1} \theta_{T-1}\right)\left(X_{T-1}+X_{T}\right)-\theta_{T-1} H_{T-1}^{*}+P_{T-1} Z_{T-1}^{*}$. It then follows from the proof of Proposition 2 that equations (25) and (26) for $t=T-1$ are indeed the solution to the optimization problem on the right-hand side of equation (23). The sum of the firm's optimal domestic currency income in periods $T-1$ and $T$, conditional on $\left(S_{1, T-2}, S_{2, T-2}\right)$, is given by

$$
\begin{aligned}
\sum_{\tau=T-1}^{T} \tilde{\Pi}_{\tau}^{*}= & S_{1, T-2} S_{2, T-2} \sum_{\tau=T-1}^{T} X_{\tau} \\
& +\sum_{\tau=T-1}^{T}\left(S_{1, T-2}+\sum_{h=0}^{\tau-(T-1)} \tilde{\theta}_{T-1+h}\right) \tilde{\epsilon}_{\tau} \sum_{h=\tau}^{T} X_{h} \\
& +\sum_{\tau=T-1}^{T}\left(2 P_{\tau}-\left|\tilde{\theta}_{\tau}\right|\right) \frac{Z_{\tau}^{*}}{2}+\sum_{\tau=T-1}^{T} \beta_{\tau} \tilde{\theta}_{\tau}^{2} \sum_{h=\tau}^{T} X_{h}
\end{aligned}
$$

where we have substituted equations (25) and (26) into equation (20) for $t=T-1$ and used equation (A-12). Thus, we have

$$
\begin{aligned}
& V_{T-1}\left(W_{T-2} \mid S_{1, T-2}, S_{2, T-2}\right)= \\
& E\left[U\left(W_{T-2}+\sum_{\tau=T-1}^{T} \tilde{\Pi}_{\tau}^{*}\right) \mid S_{1, T-2}, S_{2, T-2}\right],
\end{aligned}
$$

where $\sum_{\tau=T-1}^{T} \tilde{\Pi}_{\tau}^{*}$ is defined in equation (A-15).

Now, suppose that equations (25) and (26) describe the firm's optimal crosshedge positions from period $t+1$ to period $T$. In period $t$, the martingale assumption implies that $\Omega_{t}$ is as informative as $\left(S_{1, t-1}, S_{2, t-1}\right)$. The sum of the firm's 
optimal domestic currency income from period $t+1$ to period $T$, conditional on $\left(S_{1, t-1}, S_{2, t-1}\right)$, is given by

$$
\begin{aligned}
\sum_{\tau=t+1}^{T} \tilde{\Pi}_{\tau}^{*}= & \left(S_{1, t-1}+\tilde{\theta}_{t}\right)\left(S_{2, t-1}+\beta_{t} \tilde{\theta}_{t}+\tilde{\epsilon}_{t}\right) \sum_{\tau=t+1}^{T} X_{\tau} \\
& +\sum_{\tau=t+1}^{T}\left(S_{1, t-1}+\sum_{h=0}^{\tau-t} \tilde{\theta}_{t+h}\right) \tilde{\epsilon}_{\tau} \sum_{h=\tau}^{T} X_{h} \\
& +\sum_{\tau=t+1}^{T}\left(2 P_{\tau}-\left|\tilde{\theta}_{\tau}\right|\right) \frac{Z_{\tau}^{*}}{2}+\sum_{\tau=t+1}^{T} \beta_{\tau} \tilde{\theta}_{\tau}^{2} \sum_{h=\tau}^{T} X_{h}
\end{aligned}
$$

where we have substituted equations (25) and (26) into equation (20) recursively. The firm's decision problem as described on the right-hand side of equation (24) becomes

$$
\max _{H_{t}, Z_{t}} E\left[U\left(W_{t-1}+\tilde{\Pi}_{t}+\sum_{\tau=t+1}^{T} \tilde{\Pi}_{\tau}^{*}\right) \mid S_{1, t-1}, S_{2, t-1}\right]
$$

where $\tilde{\Pi}_{t}$ and $\sum_{\tau=t+1}^{T} \tilde{\Pi}_{\tau}^{*}$ are defined in equations (20) and (A-17), respectively. The first-order conditions for program (A-18) are given by

$$
\begin{aligned}
& E\left\{\left[1-2 b\left(W_{t-1}+\sum_{\tau=t}^{T} \tilde{\Pi}_{\tau}^{*}\right)\right] \tilde{\theta}_{t} \mid S_{1, t-1}, S_{2, t-1}\right\}=0 \\
& E\left\{\left[1-2 b\left(W_{t-1}+\sum_{\tau=t}^{T} \tilde{\Pi}_{\tau}^{*}\right)\right]\left[P_{t}-\max \left(-\tilde{\theta}_{t}, 0\right)\right] \mid S_{1, t-1}, S_{2, t-1}\right\}=0 \text {. }
\end{aligned}
$$

Note first that $\tilde{\theta}_{\tau}$ and $\tilde{\epsilon}_{\tau}$ have zero mean and are independent of each other and over time. Note also that $P_{t}=E\left[\max \left(-\tilde{\theta}_{t}, 0\right)\right]$, and $P_{\tau}$ and $Z_{\tau}^{*}$ are invariant to different realizations of $\tilde{\theta}_{t}$ for all $\tau=t+1, \ldots, T$. Thus, the above two equations reduce to

$$
\begin{aligned}
\int_{-\Theta_{t}}^{0} \Pi_{1 t}^{*}\left(\theta_{t}\right) \theta_{t} d G_{t}\left(\theta_{t}\right)+\int_{0}^{\Theta_{t}} \Pi_{2 t}^{*}\left(\theta_{t}\right) \theta_{t} d G_{t}\left(\theta_{t}\right) & =0 \\
\int_{-\Theta_{t}}^{0} \Pi_{1 t}^{*}\left(\theta_{t}\right)\left(P_{t}+\theta_{t}\right) d G_{t}\left(\theta_{t}\right)+\int_{0}^{\Theta_{t}} \Pi_{2 t}^{*}\left(\theta_{t}\right) P_{t} d G_{t}\left(\theta_{t}\right) & =0
\end{aligned}
$$

where $\Pi_{1 t}^{*}\left(\theta_{t}\right)=\Pi_{2 t}^{*}\left(\theta_{t}\right)+\theta_{t} Z_{t}^{*}$ and $\Pi_{2 t}^{*}\left(\theta_{t}\right)=\left(S_{1, t-1}+\theta_{t}\right)\left(S_{2, t-1}+\beta_{t} \theta_{t}\right) \sum_{\tau=t}^{T} X_{\tau}-$ $\theta_{t} H_{t}^{*}+P_{t} Z_{t}^{*}$. It then follows from the proof of Proposition 2 that equations (25) and (26) are indeed the solution to the optimization problem on the right-hand side of equation (24). 


\section{Journal of Financial and Quantitative Analysis}

\section{References}

$\rightarrow$ Benninga, S., and M. E. Blume. "On the Optimality of Portfolio Insurance." Journal of Finance, 40 (1985), 1341-1352.

$\rightarrow$ Black, F., and M. J. Scholes. "The Pricing of Options and Corporate Liabilities." Journal of Political Economy, 81 (1973), 637-659.

Bodnar, G. M., and G. Gebhardt. "Derivatives Usage in Risk Management by U.S. and German NonFinancial Firms: A Comparative Survey." Journal of International Financial Management and Accounting, 10 (1999), 153-187.

$\rightarrow$ Bodnar, G. M.; G. Hayt; and R. C. Marston. "1995 Wharton Survey of Derivative Usage by U.S. Non-Financial Firms.” Financial Management, 25 (1996), 113-133.

$\rightarrow$. "1998 Wharton Survey of Financial Risk Management by U.S. Non-Financial Firms.” Financial Management, 27 (1998), 70-91.

$\rightarrow$ Brennan, M. J., and R. Solanki. "Optimal Portfolio Insurance." Journal of Financial and Quantitative Analysis, 16 (1981), 279-300.

Drèze, J. H., and F. Modigliani. “Consumption Decisions under Uncertainty." Journal of Economic Theory, 5 (1972), 308-335.

$\rightarrow$ Duffie, D., and H. R. Richardson. "Mean-Variance Hedging in Continuous Time." Annals of Applied Probability, 1 (1991), 1-15.

Eiteman, D. K.; A. I. Stonehill; and M. H. Moffett. Multinational Business Finance. Massachusetts: Addison-Wesley (1998).

$\rightarrow$ Froot, K. A.; D. S. Scharfstein; and J. C. Stein. "Risk Management: Coordinating Corporate Investment and Financing Policies.” Journal of Finance, 48 (1993), 1629-1658.

Franke, G.; R. C. Stapleton; and M. G. Subrahmanyam. "Who Buys and Who Sells Options: The Role of Options in an Economy with Background Risk." Journal of Economic Theory, 82 (1998), 89-109.

Haugh, M. B., and A. W. Lo. “Asset Allocation and Derivatives.” Quantitative Finance, 1 (2001), 45-72.

Jacque, L. L. Management and Control of Foreign Exchange Risk. Boston, MA: Kluwer Academic Publishers (1996).

Kimball, M. S. "Precautionary Saving in the Small and in the Large." Econometrica, 58 (1990), 53-73.

"Standard Risk Aversion." Econometrica, 61 (1993), 589-611.

$\rightarrow$ Leland, H. E. "Saving and Uncertainty: The Precautionary Demand for Saving." Quarterly Journal of Economics, 82 (1968), 465-473.

594.

"Who Should Buy Portfolio Insurance." Journal of Finance, 35 (1980), 581-

Meese, R. "Currency Fluctuations in the Post-Bretton Woods Era." Journal of Economic Perspectives, 4 (1990), 117-134.

Rawls, S. W., and C. W. Smithson. "Strategic Risk Management." Journal of Applied Corporate Finance, 1 (1990), 6-18.

Sercu, P., and R. Uppal. International Financial Markets and the Firm. Cincinnati, OH: SouthWestern College Publishing (1995).

$\rightarrow$ Smith, C. W., and R. Stulz. "The Determinants of Firms' Hedging Policies." Journal of Financial and Quantitative Analysis, 20 (1985), 391-405.

$\rightarrow$ Stulz, R. "Optimal Hedging Policies." Journal of Financial and Quantitative Analysis, 19 (1984), 127-140. Economics, 26 (1990), 3-27.

$\rightarrow$ Tufano, P. "Who Manages Risk? An Empirical Examination of Risk Management Practices in the Gold Mining Industry." Journal of Finance, 51 (1996), 1097-1137. 